\title{
Automatic Updating of Times Remaining in Surgical Cases Using Bayesian Analysis of Historical Case Duration Data and "Instant Messaging" Updates from Anesthesia Providers
}

Franklin Dexter, $\mathrm{MD}, \mathrm{PhD}^{*}$

Richard H. Epstein, MD+

John D. Lee, PhD

Johannes Ledolter, PhD§
BACKGROUND: Operating room (OR) whiteboards (status displays) communicate times remaining for ongoing cases to perioperative stakeholders (e.g., postanesthesia care unit, anesthesiologists, holding area, and control desks). Usually, scheduled end times are shown for each OR. However, these displays are inaccurate for predicting the time that remains in a case. Once a case scheduled for $2 \mathrm{~h}$ has been on-going for $1.5 \mathrm{~h}$, the median time remaining is not $0.5 \mathrm{~h}$ but longer, and the amount longer differs among procedures.

METHODS: We derived the conditional Bayesian lower prediction bound of a case's duration, conditional on the minutes of elapsed OR time. Our derivations make use of the posterior predictive distribution of OR times following an exponential of a scaled Student $t$ distribution that depends on the scheduled OR time and several parameters calculated from historical case duration data. The statistical method was implemented using Structured Query Language (SQL) running on the anesthesia information management system (AIMS) database server. In addition, AIMS workstations were sent instant messages displaying a pop-up dialog box asking for anesthesia providers' estimates for remaining times. The dialogs caused negotiated interruptions (i.e., the anesthesia provider could reply immediately, keep the dialog displayed, or defer response). There were no announcements, education, or efforts to promote buy-in.

RESULTS: After a case had been in the OR longer than scheduled, the median remaining OR time for the case changes little over time (e.g., $35 \mathrm{~min}$ left at 2:30 PM and also at 3:00 PM while the case was still on-going). However, the remaining time differs substantially among surgeons and scheduled procedure(s) (16 min longer [10th percentile], 35 min [50th], and $86 \mathrm{~min}$ [90th]). We therefore implemented an automatic method to estimate the times remaining in cases. The system was operational for $>119$ of each day's 120 5-min intervals. When instant message dialogs appearing on AIMS workstations were used to elicit estimates of times remaining from anesthesia providers, acknowledgment was on average within $1.2 \mathrm{~min}$ (95\% confidence interval [CI] 1.1-1.3 $\mathrm{min})$. The 90th percentile of latencies was $6.5 \mathrm{~min}$ (CI: $4.4-7.0 \mathrm{~min}$ ).

CONCLUSIONS: For cases taking nearly as long as or longer than scheduled, each $1 \mathrm{~min}$ progression of OR time reduces the median time remaining in a case by $<1 \mathrm{~min}$. We implemented automated calculation of times remaining for every case at a 29 OR hospital. (Anesth Analg 2009;108:929-40) perating room (OR) whiteboards (status displays) are ubiquitous for communicating the times remaining in ongoing cases to the many stakeholders involved in perioperative patient care. ${ }^{1,2}$ Typically, the cases scheduled in each OR are represented by a linear series of bars or circles, with the OR times of completed cases determined by the actual OR times, and

From the Division of Management Consulting, Departments of Anesthesia and Health Management and Policy, University of Iowa, Iowa City, Iowa; +Department of Anesthesiology, Jefferson Medical College, Philadelphia, Pennsylvania; Departments of łMechanical and Industrial Engineering, and \$Management Sciences, University of Iowa, Iowa.

Accepted for publication October 6, 2008.

FD is the Director of the Division of Management Consulting of the Department of Anesthesia of the University of Iowa. He receives no funds personally other than his salary from the State of Iowa, including no travel expenses or honoraria, and has tenure with no incentive program. the times remaining in ongoing cases determined by the scheduled durations. ${ }^{3,4}$ Thus, a case scheduled for

Franklin Dexter is editor of Economics, Education, and Policy for the Journal. This manuscript was handled by Steven L. Shafer, Editor-in-chief, and Dr. Dexter was not involved in any way with the editorial process or decision.

Presented at the American Society of Anesthesiologists' meeting in Orlando, FL, on October 19, 2008. A presentation was also given at the Institute for Operations Research and the Management Sciences' meeting in Washington, DC, October 12, 2008.

Address correspondence and reprint requests to Franklin Dexter, $\mathrm{MD}, \mathrm{PhD}$, Division of Management Consulting, Department of Anesthesia, University of Iowa, Iowa City, IA 52242. Address e-mail to Franklin-Dexter@Ulowa.edu or go to web site www.FranklinDexter.net.

Copyright (c) 2009 International Anesthesia Research Society DOl: 10.1213/ane.0b013e3181921c37 
$2 \mathrm{~h}$ that has been in the OR for $1.5 \mathrm{~h}$ is listed as having $0.5 \mathrm{~h}$ remaining, even though most of these cases will take longer than their scheduled $2 \mathrm{~h}$. Once a case's OR time exceeds the scheduled duration, many facilities make arbitrary decisions for the display of the time remaining (e.g., $15 \mathrm{~min}$ is added to the current time whenever the screen is refreshed).

Although anesthesia providers can be called and asked for estimates of the times remaining in cases, there are limitations. Since interruptions may adversely affect OR team performance, ${ }^{5,6}$ such calls are made sparingly, resulting in updates being made infrequently. The intervals at which additional calls should be placed to update these estimates (e.g., 30 min) are unknown. The estimates often are not added to the whiteboards. Even if added, each successive 1 min the displays typically reduce $1 \mathrm{~min}$ from the estimate, and whether the estimate then remains accurate is unknown.

Video cameras in ORs can be used to transmit images that are degraded to mitigate patient or staff identification but sufficient to allow a viewer to discern progress in the case. ${ }^{7}$ However, the resulting visual information needs to be interpreted by a person knowledgeable in many types of procedures. We are not aware of those data being used for on-line decision support.

In this article, we study the statistical properties of times remaining in late running cases. We modify the previously developed Bayesian method to estimate OR times to update automatically the remaining times of ongoing cases. ${ }^{8}$ The method relies on the scheduled case durations, historical case duration data, and elapsed times determined ${ }^{9}$ by using anesthesia information management system (AIMS) data. The patient status is displayed at the anesthesia control desk and is updated automatically every $5 \mathrm{~min}$ for every case. The method is not dependent on the amount of historical data that are available, including situations where a case has never been done before by a given surgeon, and there is no requirement for human data entry. In addition, anesthesia providers are occasionally sent "instant messages" that appear on their AIMS workstation. These are transmitted via a pop-up dialog box containing a digital clock control through which they notify the OR control desk of their estimate of the time of OR exit (Fig. 1). The anesthesia provider's estimate is substituted in the Bayesian method to replace the initial (scheduled) OR time. Thus, $5 \mathrm{~min}$ later, 10 min later, etc., displays for the time remaining in the case accurately reflect the estimated time remaining $5 \mathrm{~min}, 10 \mathrm{~min}$, etc., after the provider made an estimate.

\section{METHODS}

Appendix 1 describes the mathematics programmed using structured query language (SQL Server, Microsoft, Redmond, WA). That Appendix is

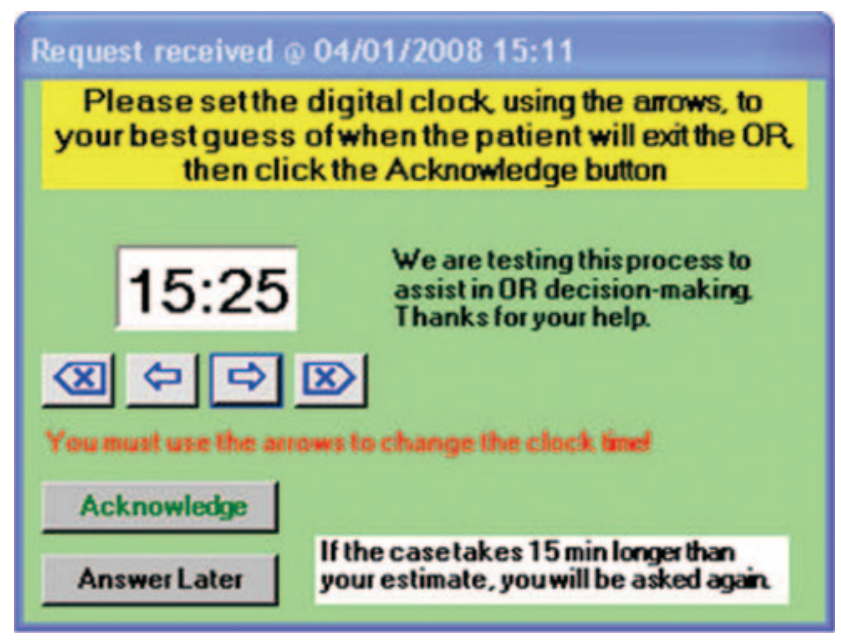

Figure 1. Unanchored version of the dialog requesting the remaining time in the case seen by anesthesia providers. The small arrows changed the clock in 5 min increments, while the large arrows made changes of $15 \mathrm{~min}$. The minimum time allowed was the current time, rounded up to the nearest $5 \mathrm{~min}$. If users attempted to enter the time directly into the digital area, this was prevented and the text in red displayed. A message was also displayed if the provider attempted to change the clock to earlier than the minimum time allowed. The red text disappeared when any arrow control was clicked subsequently.

the part of the article that describes the underlying mathematical basis of the intervention. The Appendix is presented in sufficient detail that other facilities can implement our methods.

Every $5 \mathrm{~min}$, a stored procedure on the AIMS database server calculates the elapsed time in each case from patient entry into the OR, for every case currently running in all 29 ORs of four physically distributed suites. The OR director's status display (whiteboard) and the managerial decision support system are updated automatically with these times. The time of patient entry into each OR is obtained from the AIMS (see Discussion). ${ }^{9}$ The surgeon and scheduled procedure(s) are obtained from the hospitals' operating room information management system (ORIMS). Those data are used to choose parameters from lookup tables that are regenerated using a SQL procedure that is run quarterly using all data from January 1, 2001 to the current date. The Results presented here are based on cases from 2001 to 2007 applied to forecasts for February and March 2008.

The Bayesian method to estimate OR times is applied regardless of whether the case is of a combination of surgeon and procedure(s) with no, a few, or many historical cases of the same type. ${ }^{8}$ When a case is of a rare combination with little or no historical data, ${ }^{10,11}$ the scheduled OR time is the principal or sole basis for future predictions of OR time. When a case has substantial historical data, the scheduled OR time has a negligible effect on OR time as compared to the information from the historical OR times. At the studied hospital, the schedulers add an estimate of the turnover time to the estimate of the OR time 
provided by the surgeon. Therefore, the scheduled OR time used in the Bayesian method is estimated from the scheduled case duration (see Appendix 2).

Equation 11 in Appendix 1 gives the automatically updated estimate of the time remaining in each case. However, the Bayesian method only uses data available before the case started and the automatically determined $^{7,9,12}$ time from case start. The longer that each case proceeds, the greater is the expected incremental value of the knowledge of the providers in the OR. Therefore, occasionally an instant message is sent to the AIMS workstation to elicit an update from the anesthesia provider of the remaining OR time (Fig. 1) (Appendix 3). The elicited time replaces the scheduled OR time for status displays, the decision support system, and future elicited times. Appendix 4 describes how we used the data from 560 such cases to assess the "up-time" of the system and how long anesthesia providers took to respond to the dialogs.

\section{RESULTS}

Figure 2 shows an example of how the predicted OR time remaining in cases differs among cases depending on the parameter values of the combinations of surgeon and procedure(s). Both lines are for cases with a scheduled OR time of $1 \mathrm{~h}$ and a median of historical OR time of $1 \mathrm{~h}$. After $1 \mathrm{~h}$ in the OR, the updated median time remaining for the two cases are $9 \mathrm{~min}$ and $27 \mathrm{~min}$, respectively. Later (e.g., at $1.5 \mathrm{~h}$ in the OR), the median times remaining are persistently close to a constant of $7 \mathrm{~min}(12 \%$ of $60 \mathrm{~min})(---)$ or $28 \mathrm{~min}(47 \%$ of $60 \mathrm{~min})(-)$, respectively. For the latter case (-), if the surgeon was asked at $1.5 \mathrm{~h}$ "how much longer," a likely answer would be "a half hour." At $2.0 \mathrm{~h}$, if the case were still ongoing, a likely answer would still be "a half hour." Figure 2 shows that this commonplace opinion about surgeons' updated estimates is reasonable, but that the additional OR time that is needed differs among cases. See the last three paragraphs of Appendix 2 for statistical details.

Figure 3 expands upon the result of Figure 2 by showing results for the estimated parameters of 560 cases. The cumulative frequency distribution on the left shows that, among cases that exceed the 90th percentile of their predicted OR time distribution, the median time remaining varies considerably among combinations. The 10th, 50th, and 90th percentiles are $16 \mathrm{~min}, 35 \mathrm{~min}$, and $86 \mathrm{~min}$, respectively. The cumulative frequency distribution of the times remaining each expressed as a percentage of the median of the predictive distribution is shown in the graph on the right. The 10th, 50th, and 90th percentiles are $12 \%$, $21 \%$, and $38 \%$, respectively. The importance of the heterogeneity among surgeons and procedure(s) shown by Figure 3 is that automatic estimation of remaining OR time needs to rely on historical case duration data for each combination (i.e., Eqs. 1-11). It is inaccurate to consider all cases that have exceeded their scheduled durations as having either $30 \mathrm{~min}$ or $20 \%$ of their scheduled durations as their times remaining, or any other constant for all cases at a facility.

Figure 4 shows median times remaining as a function of the elapsed time for all cases of the two most commonly scheduled durations (see caption). Analysis is nonparametric (i.e., does not rely on the Bayes method and its statistical assumptions). As each case progresses, the average time remaining declines, remains moderately constant, and then may increase. However, increases occur once cases have been ongoing so much longer than scheduled that there are few such cases. Thus, the $95 \%$ confidence intervals (CI) for the median are wide.

Every $5 \mathrm{~min}$, the SQL implementation of the Bayes approach updates the estimated times of every case at the 29 OR surgical suite that is ongoing or not yet started. The under-estimate for the up-time of this process of automatic calculation of estimated times was $99.3 \%$ (95\% CI: 98.1\%-99.9\%) ( $n=33$ days). Equivalently, for $>119$ of each day's 120 5-min intervals, the display was up to date for every case that was on-going or had not yet started. Appendix 4 has more details.

Appendix 5 describes some observations and resulting design decisions. Although anesthesia providers are physically present in ORs, can observe the actual progress of cases, and ask surgeons for feedback on times remaining, elicited estimates were only $4.7 \mathrm{~min}$ more accurate than the Bayesian method. In addition, the "instant message" dialogs are negotiated interruptions (see Appendix 4). Thus, times remaining are elicited only for cases that have exceeded their scheduled durations by more than $13 \mathrm{~min}$. Following Sandberg et al. ${ }^{13}$ in their use of AIMS prompts, we made no announcements, sought no buy-in, and performed no education about the new system. The percentiles of latencies ${ }^{14}$ to acknowledgment were 1.2 min (50th), $2.1 \mathrm{~min}$ (75th), and $6.5 \mathrm{~min}$ (90th). The corresponding 95\% CI were 1.1-1.3 $\mathrm{min}, 1.9-2.2 \mathrm{~min}$, and $4.4-7.0 \mathrm{~min}$, respectively ( $n=560$ cases).

\section{DISCUSSION}

Currently, at the studied hospital, the OR directors' status displays (whiteboard) and the managerial decision support system are updated automatically, without relying on clinicians for data entry. Every $5 \mathrm{~min}$, Bayesian estimates are recalculated for every case that has either not yet started or is on-going. When instant message dialogs (Fig. 1) are sent (e.g., if resulting decisions are ambiguous), those elicited responses replace the scheduled OR time in the Bayesian method for future whiteboard updates (i.e., if a person estimates 45 min left at 4:30 PM, the boards do not incorrectly display $15 \mathrm{~min}$ left if the case is still on-going at 5:00 PM). Figures 2-4 capture the principal results of our article, which show the need for those updates. 
Time in OR (min)
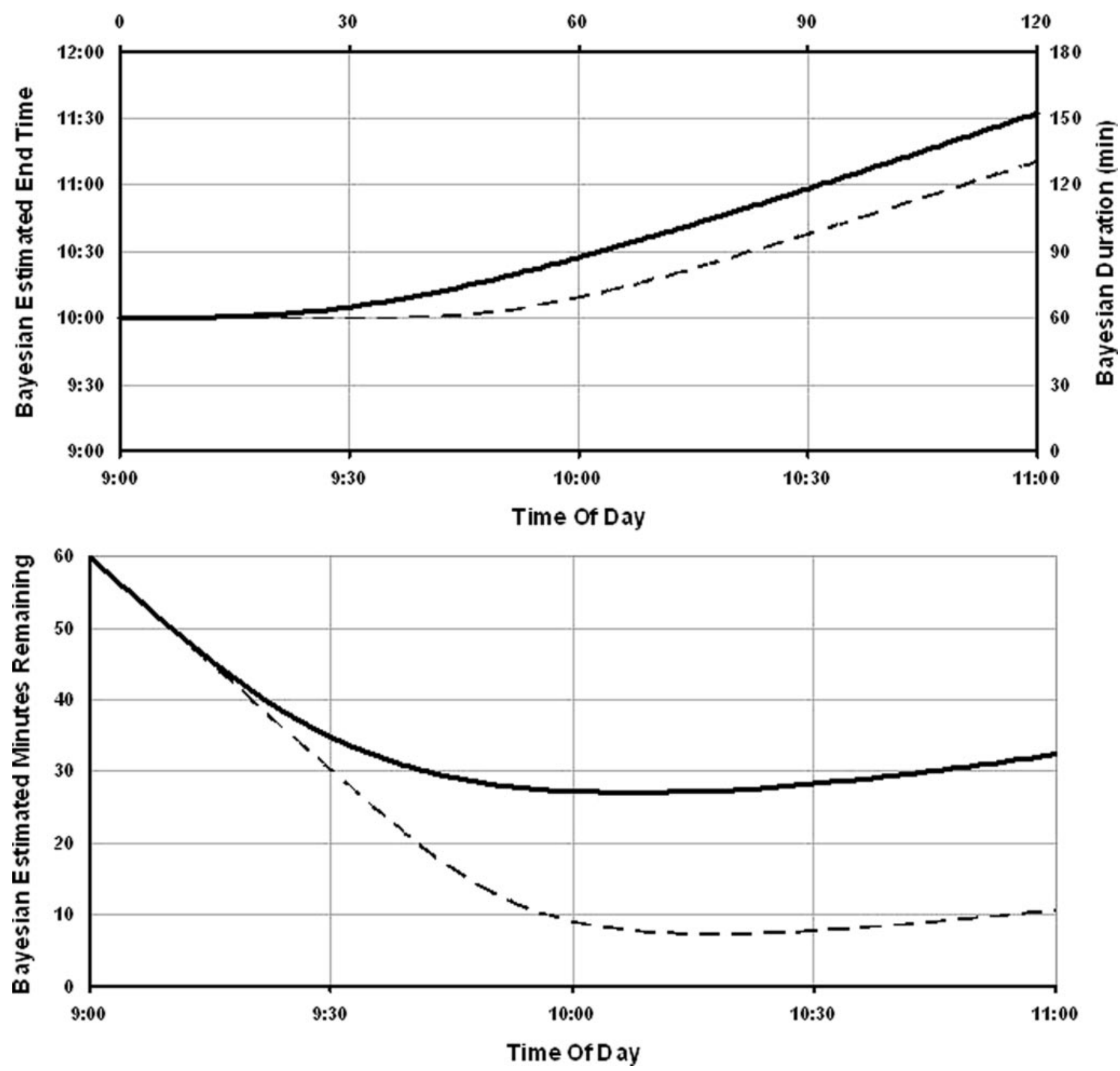

Figure 2. Example of the Bayesian updating method for two combinations of surgeon and scheduled procedures. Both hypothetical cases are scheduled to enter the OR at 9:00 AM and last for $60 \mathrm{~min}$. The estimated median end time of the cases (i.e., $d_{0.50 \mid d_{\min }}$ as calculated in Eq. 11) increase over time (i.e., $d_{\min }$ ), as the cases progress. Early in the cases, the effect is minimal, as shown at 9:15 AM, when the cases have been ongoing for $15 \mathrm{~min}$ and the estimated durations of the cases are only changed by $1 \mathrm{~min}$. However, at 10:15 AM, when the cases have been ongoing for $75 \mathrm{~min}$ (i.e., exceeding the scheduled OR time by 15 $\mathrm{min})$, the new Bayesian end times are either 10:22 (-- ) or 10:42 (-), depending on the surgeon and scheduled procedure(s). Parameter values used to create the figure are given in the third to last paragraph of Appendix 2.

Since status displays communicate ${ }^{15}$ publicly the valued $^{16}$ work of individuals and the information affects decisions (e.g., assignment of add-on cases), ${ }^{2}$ prior ethnographic results apply showing that some people manipulate ${ }^{15}$ the data displayed when given the opportunity. For example, they may indicate that the case will last longer than expected to reduce the chance of the late afternoon add-on case from the intensive care unit being assigned to their OR. Automatic updating is unbiased.
Implementation of the automatic updates of displays (whiteboards) and the decision support system relies on knowing what case are underway in each OR at all times. We automatically infer the actual location of cases based on the identifier of the AIMS workstation transmitting pulse oximetry, electrocardiogram heart rate, and end tidal $\mathrm{CO}_{2}$ partial pressures to infer the location of each case, as previously described. ${ }^{9}$ That information is used to identify the historical data for each case to apply the Bayesian method (Figs. 2 and 3). 

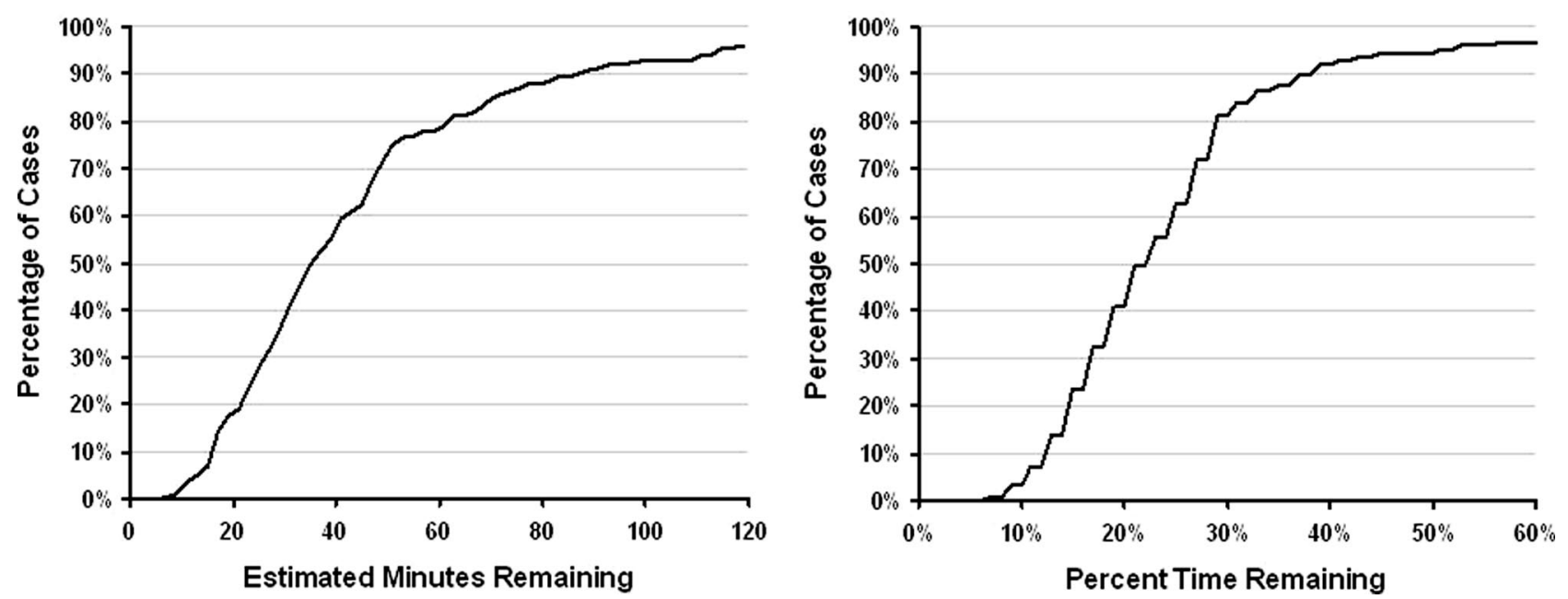

Figure 3. Comparison of automatic estimation of time remaining in cases using the Bayesian approach versus assuming results that would be obtained if the same absolute or proportional amount of time were used for all cases. The Bayesian parameters $\alpha_{k}^{*}, \beta_{k}^{*}, n_{k}$, and $\mu_{k}^{*}$ from Eqs. 2-4 were used for each of the $n=560$ cases. For each case, we calculated the time at which $90 \%$ of cases with the same scheduled OR time, surgeon and procedure(s) would be completed $\left(d_{0.90}\right.$, Eq. 6). The result was used to calculate the expected (median) time remaining $\left(d_{0.50 \mid d_{0.90}}\right.$ Eq. 11). On the left, we show the cumulative frequency distribution of the additional OR time in units of minutes: $d_{0.50 \mid d_{0.90}}-d_{0.90}$. The percentiles (with 95\% confidence intervals given in parentheses) are $16 \mathrm{~min}$ (15-17 $\mathrm{min}$ ) for the 10 th percentile, $35 \mathrm{~min}$ (33-38 min) for the 50 th, and $86 \mathrm{~min}$ (76-99 min) for the 90th. The right-hand side shows the cumulative distribution function of the difference expressed as a proportion of $d_{0.50}:\left(d_{0.50 \mid d_{0.90}}-d_{0.90}\right) / d_{0.50}$. The percentiles are $0.12(0.11-0.13)$ for the 10 th percentile, $0.21(0.20-0.22)$ for the 50 th, and 0.38 (0.34-0.41) for the 90th.

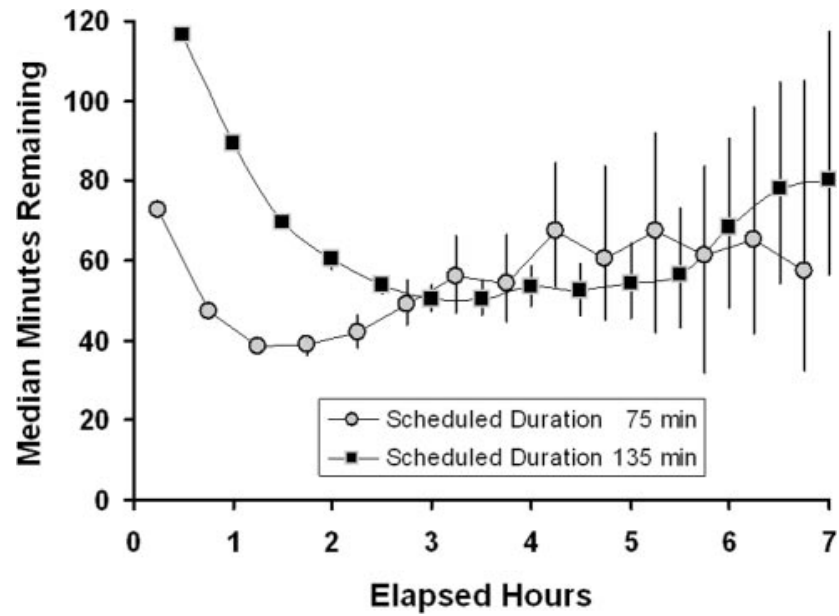

Figure 4. Median times remaining in cases as a function of the elapsed time in hours. Results are shown for the two most commonly scheduled durations at the studied hospital: $75 \mathrm{~min}$ and $135 \mathrm{~min}$. The data used were all such cases from 2001 through 2007. There are 4644 such cases for $75 \mathrm{~min}$ and 4686 for $135 \mathrm{~min}$. The figure shows that the findings of Figures 2 and 3 are not aberrancies caused by the assumptions of Eqs. 1-12. The analysis in this figure is distributionfree, with the point estimates and 95\% confidence intervals for the medians estimated using the Hodges-Lehmann method (StatXact-7).42

The statistical method is applicable for every surgical case, whether the case has 0 historical data, 99 prior data, or a value in between. That matters, because many cases at both tertiary and outpatient facilities have few or no historical data (e.g., due to rare procedure(s) and new surgeons). ${ }^{10,11,17,18}$ Furthermore, increasing the precision of case duration estimates depends on using all of the relevant data in OR information systems (e.g., not just surgeon and scheduled procedure(s), but also type of anesthetic and surgical team). ${ }^{19}$ The result of the effort to increase precision is fewer historical data per case. ${ }^{20}$

There are three principal limitations to our work.

First, recommendations from decision support systems improve decision-making on the day of surgery. ${ }^{21}$ In contrast, decisions made using status displays of ongoing OR cases, without recommendations, are correct at a rate no better than by chance alone. ${ }^{21}$ The accuracy of estimates of times remaining in cases has no substantive effect on over-utilized OR time or on overtime resulting from the decision support system's recommendations, because rarely is the inaccuracy of a sufficiently large magnitude to affect recommendations. ${ }^{1,22-24}$ Thus, how the estimates of times remaining are calculated is likely unimportant compared to the calculations being done systematically and automatically for every case. We could not, however, examine differences in resulting decisions, because anesthesia providers' estimates for time remaining are for single instants in time, whereas Bayes estimates are updated continually.

Second, the "instant message" negotiated interruptions from the dialogs and the automatic (Bayesian) methods are alternatives to using the telephone. Surgical cases studied at a facility averaged 14 and 20 interruptions per case, 1.5 by telephone. ${ }^{5,6}$ The telephone results in an immediate interruption (see Appendix 4) and its elicited time then needs to be typed into the computer for display or use in decision support. However, we do not know whether the negotiated interruptions, or 
the automatic system's elimination of interruptions, benefited OR team performance.

Third, status displays are useful not only because they contribute to decision support, but also because they facilitate asynchronous communication. $3,7,15,25$ OR coordinators use them to show the fairness of their decisions. ${ }^{25}$ Information is available so that others can provide suggestions. ${ }^{3,15}$ Staff know that supervisors and colleagues are aware of their activities. ${ }^{15}$ Anesthesiologists know when cases that they are medically directing will soon end. ${ }^{7}$ Surgeons can obtain information about when to-follow cases may start. ${ }^{7}$ However, we studied the automatic updating of displays, not how they are used. We do not know how automatic updating may be influence this use of the displays.

In summary, we successfully implemented automatic electronic whiteboard updates at a large surgical suite and have been using it to date (January 2009). We did this by deriving the Bayesian lower prediction bounds of case durations conditional on the minutes that the cases have been on-going. Every $5 \mathrm{~min}$, estimates of OR times for cases that have not yet started or are underway are updated automatically for their status displays (whiteboards) and decision support systems. The system is useful, because after a case has been in the OR substantially longer than scheduled, the median expected remaining time is relatively constant, but with the remaining time differing substantially among surgeons and scheduled procedure(s).

\section{APPENDIX 1: BAYESIAN METHOD}

Our notation matches that which we used previously to describe, ${ }^{8}$ validate, ${ }^{8}$ and apply ${ }^{8,26}$ the Bayesian method. Let the random variable $X_{k}$, refer to the natural logarithm of the OR time of a single case that is classified ${ }^{19}$ by its being of the $k$ th combination of surgeon and scheduled procedure(s), $k=1,2, \ldots, p$. For example, $k=1$ might be Dr. Smith scheduled to perform bilateral myringotomy tube placement and adenoidectomy in a child (i.e., Current Procedural Terminology-4 codes 69421 for one ear, 69421 for the other ear, and 42830 for adenoidectomy). We henceforth refer to each of the $p$ combinations as "surgeon and procedure(s)". The $n_{k}$ previously observed (historical) OR times for the $k$ th combination of surgeon and procedure(s) are $\exp \left(x_{k 1}\right), \exp \left(x_{k 2}\right), \ldots, \exp \left(x_{k n_{k}}\right)$. For example, the $n_{1}=87$ historical OR times might be $50 \mathrm{~min}, 30$ min, ..., $40 \mathrm{~min}$, resulting in the log transformed values of $x_{11}=3.9, x_{12}=3.4, \ldots, x_{1 n_{1}}=3.7$. The sample mean of the $n_{k}$ historical data $x_{k 1}, x_{k 2}, \ldots, x_{k n_{k}}$ equals $\bar{x}_{k}$ and the sample variance equals $\hat{\sigma}_{k}^{2}$. The corresponding scheduled OR times in hours are $\exp \left(x s_{k_{1} 1}\right), \exp \left(x s_{k 2}\right), \ldots, \exp \left(x s_{k n_{k}}\right)$. We assume that $X_{k}$ follows a normal distribution with an unknown mean and variance. Our objective is to predict the OR time of the next case, $\exp \left(X_{k}^{*}\right)$ based in part on its log scheduled OR time, $x s_{k}^{*}$, a constant known before the case begins. The asterisk symbol $\left(^{*}\right)$ is used to represent the next case.

The probability distribution for the next occurrence of an event after having observed historical data is called the posterior predictive distribution. We derived in Ref. 8 that the posterior predictive distribution of $X_{k}^{*}$ before the case begins follows $(\sim)$ a scaled Student $t$-distribution with $2 \alpha_{k}^{*}$ degrees of freedom:

$$
\left(X_{k}^{*}-\mu_{k}^{*}\right) \times \sqrt{\frac{\alpha_{k}^{*}}{\beta_{k}^{*}} \times \frac{\tau+n_{k}}{1+\tau+n_{k}}} \sim t\left(2 \alpha_{k}^{*}\right),
$$

where

$$
\begin{gathered}
\mu_{k}^{*}=x s_{k}^{*} \times \frac{\tau}{\tau+n_{k}}+\bar{x}_{k} \times \frac{n_{k}}{\tau+n_{k}}, \\
\alpha_{k}^{*}=\alpha+\frac{n_{k}}{2}, \\
\beta_{k}^{*}=\beta+\frac{1}{2} \sum_{i=1}^{n_{k}}\left(x_{k i}-\bar{x}_{k}\right)^{2}+\frac{n_{k} \tau\left(x s_{k}^{*}-\bar{x}_{k}\right)^{2}}{2\left(\tau+n_{k}\right)} .
\end{gathered}
$$

The constants $\alpha$ and $\beta$ are the parameters of an inverse $\gamma$ distribution that represents the prior distribution of the population variance of $X_{k}$ among the $p$ combinations of surgeon and procedure(s), ${ }^{8}$ and has previously been shown to provide a good fit (e.g., see Fig. 1 of Ref. 8). The constant $\tau$ represents the ratio of the population variance of $X_{k}$ to the variance of the prior distribution of the population mean of $X_{k}$.

Equation 2 gives the Bayesian weighted median predicted OR time of the next case. When a case is of a rare combination with little or no historical data, ${ }_{10,11}$ the scheduled OR time is the principal or sole basis for future predictions of OR time, since $\frac{\tau}{\tau+n_{k}} \cong 1$ and $\frac{n_{k}}{\tau+n_{k}} \cong 0$. When a case has substantial historical data, the scheduled OR time has a negligible effect on OR time as compared to the information from the historical OR times, since $\frac{\tau}{\tau+n_{k}} \cong 0$ and $\frac{n_{k}}{\tau+n_{k}} \cong 1$. Equation 2 shows that $\tau$ represents the number of historical cases, of a given combination of surgeon and procedure(s), for which the median predicted OR time of the next case is based equally on historical case duration data and the scheduled OR time.

The $100 \times g$ th percent lower prediction bound for the duration of each case, $d_{g}$, is given by:

$$
P\left[\exp \left(X_{k}^{*}\right) \geq d_{g}\right]=1-g,
$$

for $0<g<1$. For example, when $g=0.05$, there is a $5 \%$ chance that the OR time of the next case will be 
briefer than the value of $d_{g}$. This is known as the $5 \%$ lower prediction bound for the duration of the next case. ${ }^{2,8,27}$ From Eq. 1, this bound satisfies the expression $^{8}$ :

$$
d_{g}=\exp \left(\mu_{k}^{*}+T^{-1}\left[g, 2 \alpha_{k}^{*}\right] \times \sqrt{\frac{\beta_{k}^{*}}{\alpha_{k}^{*}} \times \frac{1+\tau+n_{k}}{\tau+n_{k}}}\right),
$$

where $T\left[., 2 \alpha_{k}^{*}\right]$ is the cumulative distribution function of the Student $t$-distribution with $2 \alpha_{k}^{*}$ degrees of freedom and $T^{-1}\left[g, 2 \alpha_{k}^{*}\right]$ is its $100 \times g$ th percentile. For example, for $g=0.05, T^{-1}\left[0.05,2 \alpha_{k}^{*}\right]=-1.65$ for large $\alpha_{k}^{*}$. When $g=0.05$, there is a $95 \%$ chance that the OR time will be at least as long as the value of Eq. 6. This lower 5\% prediction bound is almost always less than the scheduled OR time and is useful for deciding when the next patient in the same OR on the same day needs to be ready. ${ }^{2,8,28} \mathrm{~A}$ low percentage is used to mitigate delays due to patient unavailability.

We previously published an empirical study of the accuracy of Eq. $6 .{ }^{8}$ For $g=0.05$, the result $d_{0.05}$ exceeded the actual duration of $4.9 \%$ of the studied cases. ${ }^{8}$ For $g=0.90, d_{0.90}$ was exceeded by the actual duration of $9.7 \%$ of cases. ${ }^{8}$ The absolute errors of the expected values averaged $3.0 \mathrm{~min}$ less than the absolute errors of the scheduled OR times $(P<0.0001)$. The term under the square root of Eq. 6 expresses the uncertainty in the prediction. As we address at the start of the Results and at the end of Appendix 2, the term varies substantively among combinations of surgeon and procedure. ${ }^{8}$

We next expand upon our prior work. ${ }^{8}$ Consider a case that has been on-going for time $d_{\min }>0$. The $100 \times$ gth percent conditional lower prediction bound $d_{g \mid d_{\min }}$ satisfies

$$
P\left[\exp \left(X_{k}^{*}\right) \geq \mathrm{d}_{\mathrm{g} \mid \mathrm{d}_{\min }} \mid \exp \left(X_{k}^{*}\right) \geq d_{\min }\right]=1-g .
$$

From the definition of conditional probability and the fact that $d_{g \mid d_{\min }} \geq d_{\min }$, we find

$P\left[\exp \left(X_{k}^{*}\right) \geq d_{g \mid d_{\min }} \mid \exp \left(X_{k}^{*}\right) \geq \mathrm{d}_{\text {min }}\right]$

$$
=\frac{P\left[\exp \left(X_{k}^{*}\right) \geq d_{g \mid d_{\min }}\right]}{P\left[\exp \left(X_{k}^{*}\right) \geq d_{\min }\right]}
$$

Combining Eqs. 7 and 8,

$$
\begin{aligned}
P\left[\exp \left(X_{k}^{*}\right)\right. & \left.\geq d_{g \mid d_{\min }}\right] \\
& =(1-\mathrm{g}) \times P\left[\exp \left(X_{k}^{*}\right) \geq d_{\min }\right] . \\
& =(1-g) \times P\left[X_{k}^{*} \geq \ln \left(d_{\min }\right)\right] .
\end{aligned}
$$

Substituting Eq. 1 into the right-hand side,
$P\left[\exp \left(X_{k}^{*}\right) \geq d_{g \mid d_{\min }}\right]$

$$
\begin{aligned}
= & (1-g) \times P\left[\left(X_{k}^{*}-\mu_{k}^{*}\right)\right. \\
& \times \sqrt{\frac{\alpha_{k}^{*}}{\beta_{k}^{*}} \times \frac{\tau+n_{k}}{1+\tau+n_{k}}} \\
& \geq\left(\ln \left(d_{\min }\right)-\mu_{k}^{*}\right) \\
& \times \sqrt{\left.\frac{\alpha_{k}^{*}}{\beta_{k}^{*}} \times \frac{\tau+n_{k}}{1+\tau+n_{k}}\right]} \\
= & (1-g) \times\left\{1-T\left[\left(\ln \left(d_{\min }\right)-\mu_{k}^{*}\right)\right.\right. \\
& \left.\left.\times \sqrt{\frac{\alpha_{k}^{*}}{\beta_{k}^{*}} \times \frac{\tau+n_{k}}{1+\tau+n_{k}}}, 2 \alpha_{k}^{*}\right]\right\} \\
= & 1-\delta,
\end{aligned}
$$

where, ${ }^{2,28}$

$$
\delta=g+(1-g) \times p_{k \mid d_{\min }}
$$

$p_{k \mid d_{\min }}=T\left[\left(\ln \left(d_{\min }\right)-\mu_{k}^{*}\right) \times \sqrt{\frac{\alpha_{k}^{*}}{\beta_{k}^{*}} \times \frac{\tau+n_{k}}{1+\tau+n_{k}}}, 2 \alpha_{k}^{*}\right]$,

and $100 \times p_{k \mid d_{\min }}$ is the expected percentage of cases with the same parameters (Eqs. 2-4) that would have ended by time $d_{\text {min }}$. Applying Eqs. 5 and 6, the $100 \mathrm{~g}$ percent conditional lower prediction bound is given by the $100 \delta$ percent lower prediction bound for the duration of the case before it started:

$$
d_{g \mid d_{\min }}=\exp \left(\mu_{k}^{*}+T^{-1}\left[\delta, 2 \alpha_{k}^{*}\right] \times \sqrt{\frac{\beta_{k}^{*}}{\alpha_{k}^{*}} \times \frac{1+\tau+n_{k}}{\tau+n_{k}}}\right) .
$$

For example, there is a $50 \%$ chance that the OR time of a case that has been on-going for $d_{\text {min }}$ units of time will be smaller than the value $d_{0.50 \mid d_{\min }}$ in Eq. 11 .

Figure 2 shows two examples of $d_{0.50 \mid d_{\min }}$ as a function of $d_{\text {min }}$. There are four reasons why we use $g=0.50$ (i.e., the median time remaining). First, using the median minimizes the mean absolute error. ${ }^{17}$ Second, when provided anchored values, participants in experimental studies provide unbiased estimates for the median. ${ }^{29}$ Unbiased estimators have the property that the sum among cases of the differences between the actual and estimated values equals zero (i.e., there is no bias). Third, over $3 \mathrm{yr}$ at the hospital with previously published results, schedulers who were supposed to estimate the OR times of cases did so by providing unbiased estimators for the median 


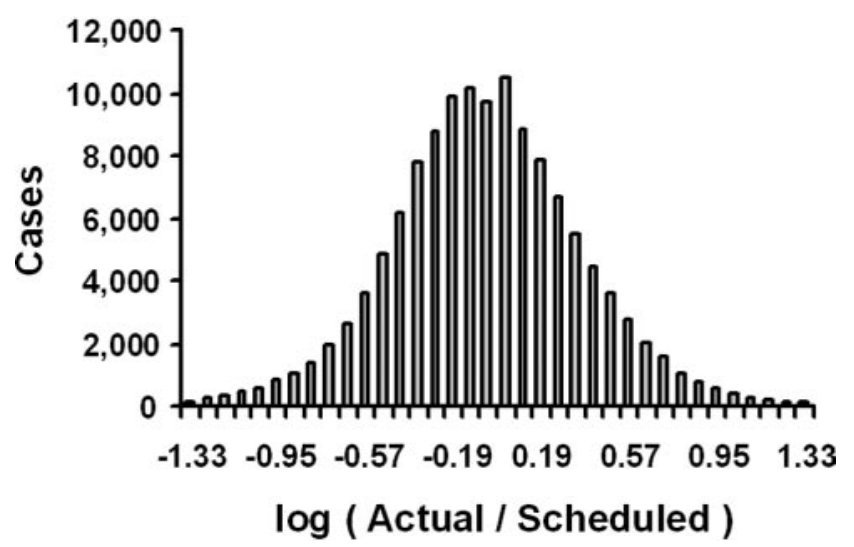

Figure 5. Histogram of the difference $\left(x_{k}^{*}-x s_{k}^{*}\right)$, where $x_{k}^{*}$ is the natural logarithm of the actual operating room (OR) time and $x s_{k}^{*}$ is the natural logarithm of the scheduled OR time. The graph is limited to the $99.0 \%$ of cases for which the differences range from $\ln (1 / 4)$ to $\ln (4)$. The graph is symmetric and centered close to zero, showing conceptually why $x s_{k}^{*}$ is a reasonable prior estimate for $x_{k}^{*}$.

OR times. ${ }^{8}$ Fourth, the method is simpler to explain because the initial estimate is $\operatorname{simply} \exp \left(\mu_{k}^{*}\right)$ from Eq. 2.

\section{APPENDIX 2: IMPLEMENTATION OF BAYESIAN METHOD}

OR information system data at the studied hospital were available from January 1, 2001 onwards. We created a lookup table of $n_{k}, \bar{x}_{k}$, and the sum of squared terms in $\beta_{k}^{*}$, while limiting consideration to the most recent 99 cases $^{27}$ of each combination of surgeon and procedure(s). However, the historical (2001-2007) raw data contained obvious errors (e.g., negatively valued or missing OR times). Therefore, when setting up the table, we excluded cases for which the actual OR time divided by the scheduled case duration was $<0.25$ or greater than 4.0. Using the 129,380 historical cases, the criteria corresponded to trimming data 3.1 and 3.3 standard deviations below and above the mean difference, respectively. The numbers of cases excluded were 416 cases $(0.3 \%)$ for missing (zero valued) actual or scheduled times, 546 cases $(0.4 \%)$ with ratios $<0.25$, and 362 cases $(0.3 \%)$ with ratios greater than 4.0 . The histogram of the difference of the logarithms of actual and scheduled OR times in Figure 5 uses $99.0 \%$ of all cases. The information from these 128,056 cases from 2001 to 2007 was used to obtain the forecasts for the Results (from February and March 2008). However, in practice, the lookup table is recalculated using a SQL server procedure that is run quarterly using all data from January 1, 2001 to the current date.

The nurse manager of the OR information management system reported that the schedulers add an estimate of the turnover time to the estimate of the OR time provided by the surgeon. If an estimated case duration is not provided by the surgeon, the scheduled duration that they use is the trimmed mean of the most recent 15 historical OR times for the surgeon performing the procedure, plus an estimated turnover. We therefore needed to analyze the resulting scheduled case durations and actual OR times to determine how to estimate scheduled OR times (i.e., time in the OR for Eq. 2) from scheduled case durations (i.e., that from the preceding paragraph). From among the 128,056 cases, there were 8045 cases $(6.3 \%)$ that were combinations of surgeon and procedure(s) observed only once (i.e., singletons) and 3370 (2.6\%) only observed twice (i.e., doubletons). The scheduled OR time was set at $96.3 \%$ of the scheduled case duration, because doing so resulted in an unbiased estimator for the 50th percentile of OR time for these 11,415 cases (for details see Limitations section of Ref. 8). We limited focus on these cases because the impact of the scheduled OR time is progressively less in Eqs. 2 and 6 for each increase in $n_{k}$. To check our use of the scheduled OR times, we then limited consideration to the 560 singletons and 470 doubletons for which other surgeons had scheduled the procedure(s) at least 30 times. The mean OR times from the other surgeons were used as the estimate of the OR time for the singletons and doubletons. ${ }^{2,30}$ The mean \pm SE of the absolute error was just $2.9 \pm 1.4 \mathrm{~min}$ less than for use of the resulting scheduled OR times. As for the previously studied hospital, use of scheduled OR times was essentially no worse than use of other surgeons' times, but offered the advantage of there being a value for every case. ${ }^{8}$

To estimate the $\alpha$ and $\beta$ in Eqs. 1-11, we followed the approach described in the Appendix of Ref. 8 The actual OR times were obtained and their logarithms were calculated for the 50,559 cases of 773 combinations of surgeon and procedure(s) with at least 30 cases. The sample variance was determined for each combination. The inverses of the 773 sample variances were fit to a $\gamma$ distribution using Systat 12 (SYSTAT Software, San Jose, CA). From the method of moments, the shape parameter was 2.33 and the scale parameter was 5.68, giving estimates $\alpha=2.33$ and $\beta=$ 0.176 . Those values are close to the $\alpha=2.32$ and $\beta=$ 0.142 published from another hospital. ${ }^{8}$

Application of the estimated $\alpha$ was an implementation challenge. The decision support system repeatedly calculates percentiles from $t$-distributions with noninteger degrees of freedom (i.e., $2 \alpha_{k}^{*}$ in Eq. 6). ${ }^{2,8-11}$ We wrote the SQL code to calculate the cumulative distribution function of the $t$-distribution using its relationship with the Incomplete Beta Function. ${ }^{31}$ The inverse is obtained iteratively using bisection over a bracketed interval from values stored in a lookup table. For example, for $g=0.26$ and $2 \alpha_{k}^{*}=5.72$, we aim to estimate $T^{-1}[0.26,5.72]$. We know a priori that $T^{-1}[0.25,5] \leq T^{-1}[0.26,5.72] \leq T^{-1}[0.30,6]$. The lower and upper bounds both are stored in the lookup table and are used as the limits of the bracketed interval. 
To estimate the $\tau$, we again used the actual and scheduled OR times for the 50,559 cases of combinations of surgeon and procedure(s) with at least 30 cases. The sample variance of the differences of the logarithms of the actual and scheduled OR times equaled 0.158 . The variance of a $t$ distribution with $2 \alpha$ degrees of freedom equals ${ }^{32} \frac{2 \alpha}{2 \alpha-2}$. Setting $n_{k}=0$ in Eq. 2, resulting in $\mu_{k}^{*}=x s_{k}^{*}$ in that equation, and substituting into Eq. 1, $\operatorname{Var}\left(X_{k}^{*}-x s_{k}^{*}\right)=\frac{\beta}{\alpha} \times \frac{\tau+1}{\tau}$ $\times \frac{2 \alpha}{2 \alpha-2}=\frac{\beta}{\alpha-1} \times \frac{\tau+1}{\tau}$. Solving the equation $\operatorname{Var}\left(X_{k}^{*}-x s_{k}^{*}\right)=0.158$ for $\tau$ leads to $\tau=5.14$ cases. In comparison, we found $\tau=8.68$ cases at the previous hospital studied. ${ }^{8}$ The accuracy of the Bayesian estimates was insensitive to these relatively small differences in the value of $\tau$ (see last section of Appendix of Ref. 8). Estimation of $\tau$ was unaffected by the proportional correction for bias, because the correction shifted only the mean of $\left(X_{k}^{*}-x s_{k}^{*}\right)$, not the $\operatorname{Var}\left(X_{k}^{*}-x s_{k}^{*}\right)$.

Figure 2 shows an example of the impact of differences in parameter values among surgeons and procedure(s) on the relationship between $d_{0.50 \mid d_{\min }}$ and $d_{\text {min }}$. For simplicity, we set $\mu_{k}^{*}=\ln (60 \mathrm{~min})$. We used $n_{k}=\tau$, and consequently $\alpha_{k}^{*}=\alpha+n_{k} / 2=2.33+5.14 / 2=$ 4.90. For $\beta_{k}^{*}$, we used the two values 0.18 and 1.27, which corresponded to the 10th and 90th percentiles of the distribution of $\beta_{k}^{*}$ for cases with $0 \leq n_{k} \leq 9$.

Figure 2 can be interpreted as a survival or reliability analysis. The bottom pane shows that after the patient has been in the OR for $\exp \left(\mu_{k}^{*}\right)$ time, the median residual lifetime is nearly constant. Let $\sigma_{k}^{*}=\sqrt{\left(\alpha_{k}^{*} / \beta_{k}^{*}\right) \times\left(\left[\tau+n_{k}\right] /\left[1+\tau+n_{k}\right]\right)}$. For $\beta_{k}^{*}=$ $0.18, \sigma_{k}^{*}=0.20$. For $\beta_{k}^{*}=1.27, \sigma_{k}^{*}=0.53$. Generally, the hazard rate of the lognormal distribution increases to a peak and then declines. ${ }^{33,34}$ However, for our range of $\sigma_{k \prime}^{*} 18$ the time of the peak in the hazard rate substantially exceeds the median OR time. ${ }^{33,34}$ Consequently, for times from $\exp \left(\mu_{k}^{*}\right)$ to $\exp \left(\mu_{k}^{*}\right)$, the hazard rate usually increases to a peak, ${ }^{34}$ and if it declines that is for a vanishingly small percentages of cases. The hazard rate can be examined using a statistics packages by generating log normally distributed random numbers with parameters $\mu_{k}^{*}$ and $\sigma_{k}^{*}$, and then applying the package's parametric (lognormal) or nonparametric survival analysis.

There were $n=560$ cases studied as part of Appendices 3 and 4 . We used the estimated posterior parameters $\left(\alpha_{k}^{*}, \beta_{k}^{*}, n_{k}\right.$, and $\left.\mu_{k}^{*}\right)$ from these cases to explore simpler (non-Bayesian) estimation of the time remaining in surgical cases (Fig. 4). In addition, we used the estimated posterior parameters to generalize Figure 2 to all cases (Fig. 3). For each case, the 90\% lower prediction bound $\left(d_{0.90}\right)$ was calculated using
Eq. 6 and then substituted into Eq. 11 to calculate the median time remaining conditional on the case already having taken as long as its $90 \%$ lower prediction bound $\left(d_{0.50 \mid d_{0.90}}\right)$. Figure 3 shows cumulative histograms reporting the difference between $d_{0.50 \mid d_{0.90}}$ and $d_{0.90}$ and the difference reported as a percentage of $d_{0.50}$.

\section{APPENDIX 3: "INSTANT MESSAGES" AND THEIR IMPLEMENTATION}

The mean time from the end of surgery to exit from the OR was determined from an analysis of the $6 \mathrm{yr}$ of historical data in the hospital's ORIMS. Conveniently, the mean was $15 \mathrm{~min}$. Every $5 \mathrm{~min}$, a SQL stored procedure calculates the elapsed time in each case in progress from patient entry into the OR. If the elapsed time exceeds the scheduled case duration plus $10 \mathrm{~min}$, and the "end of surgery" event has not been documented by the anesthesia provider, the stored procedure writes a message, addressed to the recipient workstation, to a database table. Each workstation queries the database at $1 \mathrm{~min}$ intervals and displays new messages directed to its attention using the dialog shown in Figure 1. This process resulted in a mean time from the end of surgery to the message of 12.5 $\mathrm{min}$, since the query interval was $5 \mathrm{~min}$. Because workstations queried the database for messages every $1 \mathrm{~min}$, there was an additional mean latency of 0.5 $\mathrm{min}$. Together, the earliest that the message could be sent was $10 \mathrm{~min}$ after the scheduled case duration, the latest was $16 \mathrm{~min}$, and the mean was $13 \mathrm{~min} .{ }^{14}$

When asked, respondents use a digital clock to respond with an estimate of the time when the patient will exit from the OR (Fig. 1). The clock is set to the current time plus $15 \mathrm{~min}$. Buttons are provided to adjust the clock forward or backward in 5 or $15 \mathrm{~min}$ increments, with the minimum value accepted being the current time. The properties of this dialog box are that it remains in front of all other applications, is partially transparent (i.e., objects behind the dialog are viewable), and all controls on the screen can be activated while the dialog remained displayed. The dialog included a note telling the provider: "If the case takes 15 min longer than your estimate, you will be asked again" (Fig. 1). The elicited time is sent back to the database and replaces the scheduled case duration for status displays, the decision support system, and future elicited times.

Our instant messages appeared as dialogs with forced responses (Figs. 1 and 6). Based on responses to ad hoc messages sent by the OR director using the instant messaging system, we expected that availability of a free text option would sometimes result in answers about the time of patient exit that are reported not in units of time (e.g., "as soon as the x-ray is taken for the missing sponge" instead of "2:35 $\mathrm{PM}^{\prime \prime}$ ). Thus, we do not know what would have been the 


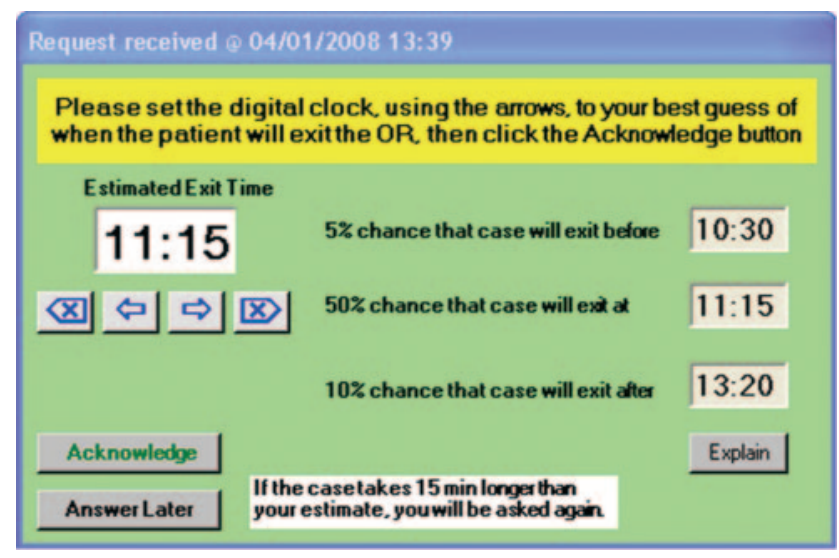

Figure 6. Anchored version of the dialog, eliciting the time remaining in the case, as seen by anesthesia providers. The features and controls are similar to those seen in Figure 1. One addition is the change in the information to the right, which provides the time anchors. The description of the objectives behind the dialog box (which was included in Fig. 1) was omitted because the staff was already familiar with this system from the previous 4 -wk period. The limits of the digital clock were anchored such that the selected time could not be earlier than the 5th percentile or later than the 90th percentile. The 90th percentile was displayed after rounding up to the next 5 $\mathrm{min}$. The 5th percentile was displayed after rounding down to the preceding $5 \mathrm{~min}$. However, since the resulting value could be sooner than the current time, the minimum value displayed for the 5th percentile was the current time rounded up to the next $5 \mathrm{~min}$. The digital clock's default value ("Estimated Exit Time") was set to the median time of OR exit as estimated by the Bayesian method and rounded down or up to the nearest 5 min. Approximately $85 \%$ of default values were changed $(95 \%$ confidence interval 79\%-90\%). The calculations for the dialogs were performed using SQL server and displayed on the workstations using VB.net (Microsoft, Redmond, WA). The figure has been altered in that what the user saw was the word "estimated" misspelled as "estimated." No users commented on the transposition, even though the AIMS administrator is often informed of other spelling mistakes in the AIMS.

perceptions of a full instant message client with twoway communication between the OR control desk and anesthesia provider, or among anesthesia providers. ${ }^{35}$

\section{APPENDIX 4: ASSESSING SYSTEM "UP TIME" AND USER RESPONSIVENESS}

We analyzed data from the dialogs after a $1 \mathrm{wk}$ test period. Approximately 75 different anesthesiology residents and certified registered nurse anesthetists were doing cases at the studied suite during the period.

First, we estimated the fraction of time that our implementation of the Bayesian method was running. The numerator was the sum of the OR times for cases with an instant message response from the dialogs of Figure 1 and the denominator was the sum of the OR times for all cases for which an instant message should have been sent. The resulting value was equivalent to the "up-time" of an e-mail server. If the up-time were $99.0 \%$, then 99 times out of 100 that you try to login to check your e-mail you would be able to do so. Our estimate for the up-time was, deliberately, an underestimate of the true up-time, because the automatic method did not actually fail for entire cases. Failure was not caused by characteristics of cases but by processes on the computer from which the queries were launched (e.g., system crashes and accidental interruptions of the program running the queries). No down time was generated by failures of the Bayesian program or running the associated queries. In addition, for the 20 workdays studied, the queries were being executed from a desktop computer being used concurrently for other work, whereas a production server is currently used. Statistical analysis was appropriate for the time series of failures. For each of the workdays, the number of whole minutes of cases with and without failure was calculated, and from that the Freeman-Tukey transformation of the proportion uptime. ${ }^{36}$ Student's $t$-distribution was used to calculate the mean and $95 \%$ CI for the 20 transformed values, and then the inverse was taken. ${ }^{37}$

Second, we analyzed the time anesthesia providers took to respond to the dialog. The interruptions caused by the dialogs are so called "negotiated" interruptions. ${ }^{38}$ This means that first the computer announces its need to interrupt the respondent, who then chooses when to deal with the interruption. Because of the special properties of the dialog, users can reply immediately, continue to work with the dialog displayed, or defer their response for $5 \mathrm{~min}$ at which time the dialog reappears (Fig. 1). Alternative methods of interruptions are "immediate" requiring response before regular work can proceed, "scheduled" that occur at prespecified times, and "mediated" that are held until the estimated workload of the respondent is low. ${ }^{38}$ We chose to use negotiated interruptions based on experimental findings that negotiated interruptions permitted the best performance at the respondent's primary work, among the different types of interruptions. ${ }^{38}$ Furthermore, the accuracy of the task initiated by a negotiated interruption was as good as for any of the other types of interruptions. ${ }^{38}$ Experimental participants said that they preferred the negotiated interruptions over both immediate and scheduled interruptions, partly based on their feeling less interrupted. ${ }^{38}$ However, these advantages were obtained at the expense of the longest latencies ${ }^{14}$ among the four different types of interruptions. ${ }^{38}$ Therefore, we developed, ${ }^{14}$ tested, ${ }^{14}$ and applied a method to examine percentiles of the times that the anesthesia providers took to reply to the dialogs, including the times of all deferrals. However, suppose that a provider receives the dialog and is about to close the anesthesia record on the workstation and leave the OR. There is no reason to reply. ${ }^{14}$ Thus, the times analyzed were the earlier of the times of exit from the OR as recorded in the AIMS and the time that the provider clicked the "Acknowledge" button (Fig. 1). The 95\% CI for the percentiles were calculated using the conservative Clopper-Pearson method. ${ }^{39,40}$ 


\section{APPENDIX 5: OBSERVATIONS AND RESULTING DESIGN DECISIONS}

Appendix 1 describes the deletion of $1.0 \%$ of cases based on $x s_{k}^{*}$ and $x_{k}^{*}$ applied to the historical data of 128,056 cases from 2001 to 2007 , not to the new data from 2008. For new cases, we considered using proactive alerts. The Bayesian method can detect whether a new case has a statistically significant $(P<0.05)$ chance of taking less or more time than scheduled ${ }^{8,26}$ :

$$
\frac{\left|x s_{k}^{*}-\mu_{k}^{*}\right|}{\sqrt{\frac{\beta_{k}^{*}}{\alpha_{k}^{*}} \times \frac{1+\tau+n_{k}}{\tau+n_{k}}}} \geq T^{-1}\left(1-0.05 / 2,2 \alpha_{k}^{*}\right) .
$$

For example, there was a $99.8 \%$ chance that the laparoscopic Nissen fundoplication scheduled for 75 min would take longer than scheduled, based on the surgeon's average of $203 \mathrm{~min}$ for the previous 97 times the procedure was scheduled. However, for only $2.7 \%$ of the 129,380 cases was Eq. 12 satisfied (i.e., aberrant scheduled durations are rare). Furthermore, among those occurrences, the $n_{k} \geq 16$ cases, the 5 th percentile of $n_{k}$ was 30 cases $(95 \%$ CI $30-30)$, and the 10 th percentile of $n_{k}$ was 41 cases (95\% CI 35-45). Thus, as for the other hospital at which we applied Eq. $12,{ }^{26}$ the $n_{k}$ sufficiently exceeded $\tau$ in Eqs. 2 and 4 that the $x s_{k}^{*}$ had negligible influence on the resulting prediction bound of Eq. 11. Therefore, our Results are based on no alerts being used about the data entered including outliers in the scheduled duration. Instead, the system simply ignores scheduled durations of $<15 \mathrm{~min}$, setting

$x s_{k}^{*}=\mu_{k}^{*}$ for $x s_{k}^{*}<\ln (15 \mathrm{~min})$.

There were $n=373$ acknowledgments from the dialog of Figure 1. We calculated the median pairwise difference between: i) the absolute error between the actual time of patient exit and Eq. 11's estimate of the median time of patient exit at the time of acknowledgment and ii) the absolute error between the actual time of patient exit and the time elicited by the dialog inquiry. The median was used, because there were some $(\cong 3 \%)$ large outliers. As described in the preceding paragraph, outliers were not excluded because they do not affect the decision support system's recommendations. The absolute error, and not the proportional error, was used because the absolute error measures the cost of the forecast error (e.g., for add-on, moving case, and relief decisions $\left.{ }^{2}\right) .{ }^{17}$ The CI for the median was calculated using the ClopperPearson method. . $^{39,40}$

Since the anesthesia providers' estimates were more accurate than the Bayesian method, we continued the use of the dialogs, despite the resulting interruption. However, because the difference was only $4.7 \mathrm{~min}$ ( $1.8 \%$ of median OR time, $95 \%$ CI: $2.0-9.4$ min) we limited use of the instant messages to cases exceeding the scheduled duration plus $13 \mathrm{~min}$ (see
Appendix 3). If the studied hospital had been classifying its cases not just based on surgeon and scheduled procedure(s), but also based on type of anesthetic and surgical team, ${ }^{19,20,41}$ likely the Bayesian method would have been more accurate.

Spearman rank correlation was tested between the proportion of like cases completed at the acknowledged time $\left(p_{k \mid d_{\min }}\right.$ Eq. 10$)$ and the case's difference between the absolute errors. The $\mathrm{CI}$ for the rank correlation was calculated from the 373 paired values. There was no significant association between i) the difference in absolute errors and ii) the number of historical OR times (Spearman $r=0.09,95 \%$ CI -0.09 to 0.27$)$. Thus, we based use of the instant messages solely on exceedance of the scheduled duration plus 13 min (see Appendix 3), not on characteristics of the case's estimated parameters.

We used least absolute values regression to estimate the linear combination of intercept, Bayesian estimate, and elicited time remaining that minimized the absolute error in OR time remaining from the time of acknowledgment (Systat 12, simplex method). The use of least absolute values regression matches the rationale described two paragraphs above. The $95 \%$ CI of parameters were consistent with a model of the estimate being $1 / 3$ rd Bayesian $+2 / 3$ rd elicited time. There was only a 0.6 min reduction in error achieved by calculated a weighted combination of the elicited time and Bayesian estimate $(0.2 \%$ of median OR time, $95 \%$ CI -0.4 to 1.5 min). Thus, we decided that each elicited time would fully replace its case's (original) scheduled OR time.

The median pairwise difference in absolute error was calculated between the regression estimate and that of elicited time alone. The estimated proportions of cases of the same combination of surgeon and procedure(s) that were expected to have finished before the times of acknowledgment were 0.47 (25th percentile), 0.65 (50th), and 0.80 (75th), respectively. There was no pairwise association between i) the proportions of like cases that would be finished by the time of acknowledgment and ii) the difference in absolute errors (Spearman $r=0.03,95 \%$ CI -0.07 to $0.13, n=373)$.

For the last $2 \mathrm{wk}$ of the trial period ( $n=187$ cases), we tried to elicit more accurate estimates by using what we thought was a more sophisticated dialog (Fig. 6). The new display included Bayesian estimates ${ }^{8}$ for the 5th and 90th percentiles of the time of OR exit from equation. These were obtained, respectively, by setting $g=0.05$ and $g=0.90$ in Eq. 11. The limits of the digital clock were anchored such that the selected time could not be earlier than the 5th percentile or later than the 90th percentile, with times selectable to the nearest $5 \mathrm{~min}$. The clock's default value was set equal to the $g=0.50$ Bayesian estimate of the time of OR exit. We calculated the Hodges-Lehmann estimate for the median difference in the anesthesia provider's absolute errors with (Fig. 6) versus without (Fig. 1) the 
anchors (StatXact-7, Cytel Software Corporation, Cambridge, MA). ${ }^{42}$ There was a trend toward larger absolute errors as when compared with the use of unanchored dialogs (difference $1.3 \mathrm{~min}, 95 \% \mathrm{CI}-1.6$ to $4.1 \mathrm{~min}$ ). Being a process improvement project, we promptly returned to the use of Figure 1. Perhaps future experimental studies with OR management displays $^{21,43}$ could omit the default value or omit the anchors while keeping the provided information.

\section{REFERENCES}

1. Dexter F, Macario A, O'Neill L. A strategy for deciding operating room assignments for second-shift anesthetists. Anesth Analg 1999;89:920-4

2. Dexter F, Epstein RD, Traub RD, Xiao Y. Making management decisions on the day of surgery based on operating room efficiency and patient waiting times. Anesthesiology 2004; 101:1444-53

3. Nemeth C, O'Connor M, Klock PA, Cook R. Discovering healthcare cognition: the use of cognitive artifacts to reveal cognitive work. Organ Stud 2006;27:1011-35

4. Xiao Y, Strader M, Hu P, Wasei M, Wieringa P. Visualization techniques for collaborative trajectory management. ACM Conference on Human Factors in Computing Systems 2006;1547-52

5. Healey AN, Sevdalis N, Vincent CA. Measuring intra-operative interference from distraction and interruption observed in the operating theatre. Ergonomics 2006;49:589-604

6. Healey AN, Primus CP, Koutantji M. Quantifying distraction and interruption in urological surgery. Qual Saf Health Care 2007;16:135-9

7. Xiao Y, Dexter F, Hu P, Dutton RP. Usage of distributed displays of operating room video when real-time occupancy status was available. Anesth Analg 2008;106:554-60

8. Dexter F, Ledolter J. Bayesian prediction bounds and comparisons of operating room times even for procedures with few or no historical data. Anesthesiology 2005;103:1259-67

9. Epstein RH, Dexter F, Piotrowski E. Automated correction of room location errors in anesthesia information management systems. Anesth Analg 2008;107:965-71

10. Dexter F, Macario A. What is the relative frequency of uncommon ambulatory surgery procedures in the United States with an anesthesia provider? Anesth Analg 2000;90:1343-7

11. Dexter F, Traub RD, Fleisher LA, Rock P. What sample sizes are required for pooling surgical case durations among facilities to decrease the incidence of procedures with little historical data? Anesthesiology 2002;96:1230-6

12. Xiao Y, Hu P, Hao H, Ho D, Dexter F, Mackenzie CF, Seagull FJ, Dutton R. Algorithm for processing vital sign monitoring data to remotely identify operating room occupancy in real-time. Anesth Analg 2005;101:823-9

13. Sandberg WS, Sandberg EH, Seim AR, Anupama S, Ehrenfeld JM, Spring SF, Walsh JL. Real-time checking of electronic anesthesia records for documentation errors and automatically text messaging clinicians improves quality of documentation. Anesth Analg 2008;106:192-201

14. Epstein RH, Dexter F, Ehrenfeld JM, Sandberg WS. Implications of event entry latency on anesthesia information management system decision support systems. Anesth Analg 2009;108:941-7

15. Riley R, Forsyth R, Manias E, Iedema R. Whiteboards: mediating professional tensions in clinical practice. Commun Med 2007;4:165-75

16. Dexter F, Lee JD, Dow AJ, Lubarsky DA. A psychological basis for anesthesiologists' operating room managerial decisionmaking on the day of surgery. Anesth Analg 2007;105:430-4

17. Dexter F, Traub RD, Qian F. Comparison of statistical methods to predict the time to complete a series of surgical cases. J Clin Monit Comput 1999;15:45-51

18. Zhou J, Dexter F, Macario A, Lubarsky DA. Relying solely on historical surgical times to estimate accurately future surgical times is unlikely to reduce the average length of time cases finish late. J Clin Anesth 1999;11:601-5
19. Strum DP, Sampson AR, May JH, Vargas LG. Surgeon and type of anesthesia predict variability in surgical procedure times. Anesthesiology 2000;92:1454-67

20. Dexter F, Dexter EU, Masursky D, Nussmeier NA. Systematic review of general thoracic surgery articles to identify predictors of operating room case durations. Anesth Analg 2008;106:1232-41

21. Dexter F, Willemsen-Dunlap A, Lee JD. Operating room managerial decision-making on the day of surgery with and without computer recommendations and status displays. Anesth Analg 2007;105:419-29

22. Dexter F, Macario A, Traub RD. Which algorithm for scheduling add-on elective cases maximizes operating room utilization? Use of bin packing algorithms and fuzzy constraints in operating room management. Anesthesiology 1999;91:1491-500

23. Dexter F, Traub RD. How to schedule elective surgical cases into specific operating rooms to maximize the efficiency of use of operating room time. Anesth Analg 2002;94:933-42

24. McIntosh C, Dexter F, Epstein RH. Impact of service-specific staffing, case scheduling, turnovers, and first-case starts on anesthesia group and operating room productivity: tutorial using data from an Australian hospital. Anesth Analg 2006; 103:1499-516

25. Xiao Y, Kiesler S, Mackenzie CF, Kobayashi M, Plasters C, Seagull FJ, Fussell S. Negotiation and conflict in large scale collaboration: a preliminary field study. Cogn Tech Work 2007;9:171-6

26. Dexter F, Macario A, Ledolter J. Identification of systematic under-estimation (bias) of case durations during case scheduling would not markedly reduce over-utilized operating room time. J Clin Anesth 2007;19:198-203

27. Wachtel RE, Dexter F. Simple method for deciding what time patients should be ready on the day of surgery without procedure-specific data. Anesth Analg 2007;105:127-40

28. Dexter F, Traub RD. Statistical method for predicting when patients should be ready on the day of surgery. Anesthesiology 2000;93:1107-14

29. Peterson C, Miller A. Mode, median, and mean as optimal strategies. J Exp Psychol 1964;68:363-7

30. Macario A, Dexter F. Estimating the duration of a case when the surgeon has not recently performed the procedure at the surgical suite. Anesth Analg 1999;89:1241-5

31. Press WH, Teukolsky SA, Vetterling WT, Flannery BP. Numerical recipes in FORTRAN. The Art of scientific computing. 2nd ed. New York: Cambridge University Press, 1992:207,220-222

32. Johnson NL, Kotz S, Balakrishnan N. Continuous univariate distributions. Vol 2. 2nd ed. New York: John Wiley \& Sons, 1995:366

33. Chang DS. Critical time of the lognormal distribution. Microelectron Reliab 1994;34:261-6

34. Chang DS. Optimal burn-in decision for products with an unimodal failure rate function. Eur J Oper Res 2000;126:534-40

35. Garrett RK, Danziger JN. IM=Interruption management? Instant messaging and disruption in the workplace. J Comput Mediat Commun 2007;13:article 2

36. Mosteller F, Youtz C. Tables of the Freeman-Tukey transformations for the binomial and Poisson distributions. Biometrika 1961;48:433-40

37. Dexter F, Marcon E, Epstein RH, Ledolter J. Validation of statistical methods to compare cancellation rates on the day of surgery. Anesth Analg 2005;101:465-73

38. McFarlane DC. Comparison of four primary methods for coordinating the interruption of people in human-computer interaction. Hum Comput Interact 2002;17:63-139

39. Clopper CJ, Pearson ES. The use of confidence or fiducial limits illustrated in the case of the binomial. Biometrika 1934;26:404-13

40. Hahn GJ, Meeker WQ. Statistical intervals. A guide for practitioners. New York: Wiley, 1991:82-4,100-5

41. Combes C, Meskens N, Rivat C, Vandamme JP. Using a KDD process to forecast the duration of surgery. Int J Prod Econ 2008;112:279-93

42. Maritz JS, Wu M, Stuadte RG Jr. A location estimator based on a U-statistic. Ann Stat 1977;5:779-86

43. Dexter F, Xiao Y, Dow AJ, Strader MM, Ho D, Wachtel RE. Coordination of appointments for anesthesia care outside of operating rooms using an enterprise-wide scheduling system. Anesth Analg 2007;105:1701-10 